\title{
Failure to Pace
}

\author{
Minati Choudhury, Kalpna Irpachi \\ Department of Cardiac Anesthesia, Cardiothoracic Sciences Centre, All India institute of medical \\ sciences, New Delhi, India-110029; E-mail: minati.2002@gmail.com
}

4 month old female child diagnosed a case of long QT syndrome with recurrent polymorphic ventricular tachycardia (VT) storm with moderate left ventricular (LV) dysfunction with status epilepticus presented with recurrent seizures in an emergency. Patient was intubated and put on mechanical ventilation. Recurrent seizures subsided with intravenous midazolam and anticonvulsants. In view of unstable rhythm, recurrent polymorphic VT storm and unstable haemodynamic, patient required antiarrhythmic agents such as flecainide and multiple inotropic supports. There was frequent pause dependent VT hence it was decided to put on permanent pacemaker. A repeated pacing attempt failed and on table plan for cardiac sympathectomy was made. However, the parents refused for the later. The patient left back with her own rhythm and parents are prognosticated.

\section{Discussion}

Patients with Long QT syndrome are prone to life threatening arrhythmias. ${ }^{1}$ After surviving such an event, implantation of an implantable cardio-verter defibrillator (ICD) is indicated. There are, however, special subtle demands in the treatment of these patients. In this case we describe our experience in a patient with Long QT syndrome who failed to pace. Such patients could always receive an atrial pacing or dual chamber device (AAI or DDD/ICD) (Level of Evidence: C) ${ }^{2}$

ThelongQT interval syndrome can lead to episodicVT, bradycardia-dependent torsades de pointes causing presyncope, syncope, seizures or cardiac arrest. Brain becomes increasingly deprived of oxygen due to erratically beating of heart, which causes seizures. ${ }^{3}$ While a pacemaker may be beneficial in patients who have recurrent episodic torsades de pointes due to bradycardia; it is recognized that ICD therapy might be recommended in symptomatic or high-risk long QT syndrome patients. For instance, a singlechamber ICD may be preferred in some situations, especially in children and adolescents, to minimize lead complications and maximize device longevity. Unfortunately, the literature regarding the benefits of pacing and selection of pacing mode in this syndrome is very limited. In one study of eight patients, pacing was instituted in three who were unsuccessfully treated with both beta blockers and left cardiothoracic sympathectomy, and in two who proved refractory or intolerant to beta-blockers. After pacing using DDD, AAI, or VVI mode with rate $70-85 \mathrm{bpm}$, there was no change in the corrected QT interval, but the measured QT interval decreased significantly. In long-term follow-up, all patients were alive and syncope-free. One patient with an AAI pacemaker developed dizziness due to AV block but remained asymptomatic after DDDR pacing. ${ }^{4}$

From an international prospective study of long QT syndrome patients, 30 patients were identified who had undergone permanent pacemaker implantation (AAI, VVI, or DDD) for the management of recurrent syncope. Though pacing reduced the rate of recurrent syncopal events in high-risk long QT syndrome patients, it did not provide complete protection with recurrent syncope or ventricular arrhythmias occurring in 9 patients. The effect of pacing on repolarization was evaluated in 10 patients in whom the demand atrial pacing rate was faster than the intrinsic rate, and a significant reduction in QT interval with a nonsignificant reduction in corrected QT interval was noted. ${ }^{5}$

Another study suggested that combined beta-blocker therapy and pacing (DDD, AAI, or VVI) at a rate designed to normalize the QT interval appeared effective for symptomatic patients with long QT syndrome, although one sudden death occurred in a patient who had discontinued beta-blocker therapy. ${ }^{2}$ Atrial pacing alone may be effective as it prevents bradycardia that causes torsades de pointes VT, and since most of these individuals have normal AV conduction, they do not require ventricular pacing. No randomized studies have compared the efficacy of a specific pacing mode for long QT syndrome. A dual-chamber pacemaker in this population may help detect episodes of VT with device monitoring that might impact patient management. It is possible that ventricular pacing in this population may lead to an increased risk of abnormal ventricular repolarization that could increase the risk for torsades de pointes VT.

The long QT syndrome is an arrhythmogenic, familial 
correspondence

disease with high risk of sudden cardiac death due to torsade de pointes and ventricular fibrillation. Cardiac pacing is indicated in patients with coincidence of AV block or evidence of symptomatic bradycardia (spontaneous or due to beta-blocking therapy) or pause-dependent ventricular tachycardia.

After pacemaker implantation beta-blockers should be continued to prevent sudden death.

Underlying prolonged myocardial refractoriness in long QT syndrome results in failure to capture at times. This happens especially if pacing impulses are delivered during the longer native refractory periods or use of antiarrythmic agents such as flecainide and amiodarone. The later may be the contributing factor to failure to pace in our patient.

Long QT syndrome with relative bradycardia attributable to $2: 1 \mathrm{AVB}$ has a poor prognosis during the first month of life. Lupoglazof et al analysed the characteristics and outcomes of 23 neonate pro-bands from Long QT syndrome population. In their study, three infants with 2:1 AVB died before one month of age: one case of sepsis in a child with a pacemaker; one case after interruption of pacing due to a loss of capture; and the last case in the absence of treatment because of parent refusal. ${ }^{6}$

Failure to pace without any provoking agent though extremely rare can happen. Left cardiac sympathectomy though not cent percent effective is the last option for selected cases.

\section{REFERENCES}

1. Gillis $A M$, Russo $A M$, Ellenbogen $K A$, Swerdlow $C D$, Olshansky B, Al-Khatib SM, et al. HRS/ACCF expert consensus statement on pacemaker device and mode selection. Heart Rhythm. 2012;9(8):1344-65. [PubMed] DOl: 10.1016/j.hrthm.2012.06.026

2. Dorostkar PC, Eldar M, Belhassen B, Scheinman MM. Long-term follow-up of patients with long-QT syndrome treated with beta-blocker and continuous pacing. Circulation. 1999;100(24):2431-6. [PubMed]

3. Lupoglazoff JM, Denjoy I, Villain E, Fressart V, Simon F, Bozio $A$, et al. Long QT syndrome in neonates: conduction disorders associated with HERG mutations and sinus bradycardia with KCNQ1 mutations. J Am Coll Cardiol. 2004;43(5):826-30. [PubMed] DOI: 10.1016/j.jacc.2003.09.049

4. Eldar M, Griffin JC, Abbott JA, Benditt D, Bhandari A, Herre $\mathrm{JM}$, et al. Permanent cardiac pacing in patients with the long QT syndrome. J Am Coll Cardiol. 1987;10(3):600-7. [PubMed]

5. Moss AJ1, Liu JE, Gottlieb S, Locati EH, Schwartz PJ, Robinson JL. Efficacy of permanent pacing in the management of high-risk patients with long QT syndrome. Circulation. 1991;84(4):1524-9. [PubMed]

6. Lupoglazoff JM, Cheav T, Baroudi G, Berthet M, Denjoy I, Cauchemez B, et al. Homozygous SCN5A mutation in longQT syndrome with functional two-to-one atrioventricular block. Circ Res. 2001;89(2):E16-21. [PubMed]

\section{Ronald Melzack Lecture Award}

Award includes a plaque to be presented at the Congress, and travel to the Congress (discount airfare), Congress registration, and accommodation expenses

\section{Guidelines:}

The Ronald Melzack Lecture Award was created in 2010 by the IASP Council in honor of IASP Honorary Member Ronald Melzack, for his work and contributions to the science of pain. The recipient is selected by the Scientific Program Committee from among plenary presentations chosen for the Congress. Funding for the award is provided by the Louise and Alan Edwards Foundation. 